\title{
VISUAL OUTCOME OF CHILDHOOD ANTERIOR PERFORATING EYE INJURIES: PROGNOSTIC INDICATORS
}

\author{
R. J. BAXTER, P. R. HODGKINS, I. CALDER, A. J. MORRELL, S. VARDY and A. R. ELKINGTON \\ Birmingham, Coventry and Southampton
}

\begin{abstract}
SUMMARY
Ninety-six cases of anterior globe perforation in children less than 16 years old, requiring surgical repair, were reviewed. Seventy-three patients were male and 23 female, with a mean age of 9 years 4 months. Perforation involving just the cornea was associated with a good visual prognosis unless: it involved the visual axis, resulting in corneal scarring and/or significant astigmatism (>3.0 DS); it involved greater than one-quarter of corneal diameter; or there was non-compliance with spectacles/patching in patients younger than 8 years old. Injuries involving the lens in those younger than 8 years were associated with a far worse prognosis due to the problems of correcting aphakia. Surgical delay and method of injury did not affect prognosis. Some patients fail to re-attend and greater patient/parent education prior to discharge is recommended.
\end{abstract}

Perforation of the eye is a frequent cause of unilateral visual loss in children, ${ }^{1-3}$ with up to $50 \%$ of all penetrating injuries occurring in that age group. ${ }^{3.5}$ The development of bandage contact lenses, tissue adhesives and other nonsurgical techniques has had a considerable impact on corneal wound management, although many cases still require surgical repair.

Children under 8 years of age are at particular risk from perforation because of the threat of amblyopia. In addition the eye may become disfigured from corneal scarring and a squint may develop. The aetiology of these injuries is also changing, far fewer resulting from road traffic accidents but substantial numbers occurring in domestic settings. ${ }^{6}$

The purpose of this study was to analyse a series of patients aged 16 years or younger who presented with perforating eye injuries localised to the anterior segment that required surgical intervention. The final visual outcome

Correspondence to: Mr P. Hodgkins, Department of Ophthalmology, Clinic 3 (Box 41), Addenbrooke's Hospital, Hills Road, Cambridge CB2 2SP, UK. and prognosis were evaluated with regard to the age, severity and location of the wound in an attempt to find factors that influence the visual prognosis.

\section{SUBJECTS AND METHODS}

The particulars of all children under 16 years of age who had a perforating ocular injury and attended the Birmingham and Midland Eye Hospital for primary repair between September 1979 and August 1989 inclusive, were reviewed. Case notes were not available on 2 patients and in total 143 notes were reviewed. The following details were recorded for each case: patient's sex and age; aetiology of injury; extent of injury; length of time prior to surgery; pre-treatment visual acuity; post-treatment visual acuity; optical correction; complications; and length of follow-up achieved. Classification of the injuries is from Eagling: ${ }^{4}$

Grade 1: Laceration of the cornea/anterior sclera with or without prolapse of the iris.

Grade 2: Wounds to the anterior segment that have also involved the lens.

Grade 3: Posterior segment perforations. No involvement of the cornea or lens.

Grade 4: Anterior and posterior segment damage.

For analysis of results the cornea was divided into a central $3 \mathrm{~mm}$ area, designated zone 1 and the remaining area of cornea designated zone 2. Wounds were classified as zone 1 if any part of them was in the central zone $3 \mathrm{~mm}$ in diameter.

In all, 96 of the 143 cases reviewed fulfilled the criteria of grade 1 or 2 injuries and were included in this study. These grades of injury were further subdivided by age, into patients $0-8$ years old and patients 9-16 years old inclusive. Grade 3 and 4 injuries were not analysed for this study because the management of posterior segment trauma has evolved greatly during the time of review, invalidating any results from this series. 


\section{RESULTS}

In total 143 cases were reviewed, 96 of which were wounds classified as grade 1 or 2 and were included in this study. Of these patients 73 were male and 23 female, a ratio of 3.2:1. The mean age of these children was 9 years 4 months. All procedures were performed under the operating microscope. Prolapsed iris was reposited if it appeared healthy; if it appeared atonic or was badly damaged it was abscised. In some cases during the primary procedure all lens matter was aspirated or complete lensectomy was performed after primary closure of the corneal laceration.

The post-operative visual acuity used in this report is the final recorded visual acuity, following post-trauma prescription of spectacles and occlusion therapy where appropriate. In the younger group with grade 1 injury, 18 of 27 patients were prescribed spectacles and 20 of 27 received occlusion, while of those with grade 2 injury, 1 of those that remained phakic required patching in addition to the other 24 aphakic children.

There were 54 grade 1 injuries ( 27 in each age group) and 42 grade 2 injuries ( 18 children aged $0-8$ years and 24 aged 9-16 years) (Table I). In 23 children, 10 with grade 1 injury and 13 grade 2 injury, it was not possible to record a pre-operative visual acuity. All of these children were less than 3 years old with the exception of 1 who was aged 5 years at the time of injury.

The time between the initial injury and surgery was 1-216 hours (mean 32 hours) for grade 1 injuries and 2-120 hours (mean 23 hours) for grade 2 injuries. The delay was in the main due to the late presentation of the child to hospital after the initial injury. The length of time prior to surgery appeared to make no difference to the final visual outcome.

The aetiology of the injuries is recorded in Table II. No association was found between aetiology of the injury and visual outcome.

\section{Grade 1}

In the younger group there was a return of vision to $6 / 18$ or better in all but $8(30 \%)$ cases (Table III): Five of these cases were due to a corneal scar in zone 1 and associated astigmatism; 2 were due to high astigmatism (6 DC, 9 DC) and had long wounds extending to the limbus; and in one 3-year-old girl with a reluctance to wear spectacles, despite only a 2 DC astigmatism with a clear axis amblyopia developed. A visual acuity of $6 / 6$ was achieved by 10

Table I. Grade 1 and 2 injuries: age and sex of patients

\begin{tabular}{lccc}
\hline & Grade $1(n=54)$ & Grade $2(n=42)$ & Total $(n=96)$ \\
\hline $\begin{array}{l}\text { 0-8 years old } \\
\text { Male }\end{array}$ & $20(37 \%)$ & $13(31 \%)$ & $33(34 \%)$ \\
Female & $7(13 \%)$ & $5(12 \%)$ & $12(13 \%)$ \\
$\quad$ Total & $27(50 \%)$ & $18(43 \%)$ & $45(47 \%)$ \\
9-16 years old & & & \\
Male & $20(37 \%)$ & $20(37 \%)$ & $40(42 \%)$ \\
Female & $7(13 \%)$ & $4(10 \%)$ & $11(11 \%)$ \\
$\quad$ Total & $27(50 \%)$ & $24(57 \%)$ & $51(53 \%)$ \\
\hline
\end{tabular}

patients $(37 \%)$. The older age group achieved $6 / 18$ or better in all but $4(15 \%)$ cases due to central corneal scarring and astigmatism. In this group 17 patients (63\%) achieved a visual acuity of $6 / 6$.

Among those with grade 1 injuries $(n=54), 18(33 \%)$ children had lacerations in zone 1 and $10(56 \%)$ achieved $6 / 12$ vision or better, with a mean astigmatism of 2.0 DC. Of the remaining $8(44 \%)$ patients who achieved a visual acuity of $6 / 18$ or worse, $6(75 \%)$ had a mean cylinder of 3.5 DC (SD 2.1) while 2 were lost to follow-up. The mean cylinder in those cases where perforation was away from the visual axis $(n=36)$ was $1.5 \mathrm{DC}(\mathrm{SD} 0.9)$ with a visual acuity of $6 / 9$ or better in 26 cases. Two patients had a deterioration from their pre-operative acuity of 1 line due to induced astigmatism.

\section{Grade 2 (Table IV)}

In the younger age group $44 \%(n=8)$ of children failed to achieve a vision of $6 / 18$ or better due to: clinical cystoid macula oedema in 1 case following intraocular lens implantation; central corneal scarring with astigmatism and aphakia in 3 cases and aphakic ambyopia in 4 cases. In the older age group $20 \%(n=5)$ failed to achieve a visual acuity of $6 / 18$ or better: 1 developed cystoid macula oedema following intraocular lens implantation; 1 developed a macular scar after the injury, 2 had significant corneal scarring with astigmatism and 1 had a subluxed lens with significant astigmatism.

There were 18 children aged $0-8$ years. Of these, 3 remained phakic with stable lens opacity and all achieved 6/9 vision; 12 were rendered aphakic and were corrected with a contact lens (only 1 achieved 6/9); and 3 children had an intraocular lens implant, 2 achieving 6/9 and 1 achieving 6/60 due to the development of cystoid macula oedema. Of these children 6 underwent lens surgery at the time of repair. Six underwent secondary lens surgery on average 6 days later and 2 underwent lensectomy 5 months after the initial repair because significant lens opacities had devèloped. With the exception of one 7-year-old all the children received their optical correction within 1 month, the average being 12 days.

There were 24 children aged 9-16 years. Of these, 3 remained phakic, 6 patients underwent primary lens removal and 15 underwent secondary lens removal. Of the 21 aphakic children 5 had an intraocular lens inserted and 16 were corrected with a contact lens. Secondary lens surgery was carried out on average 12 days after the primary

Table II. Aetiology of grade 1 and 2 perforating ocular injury

\begin{tabular}{lccc}
\hline Aetiology & Grade 1 & Grade 2 & Total \\
\hline Domestic & 16 & 12 & 28 \\
At play & 16 & 7 & 23 \\
Sports & 11 & 7 & 18 \\
School & 2 & 6 & 8 \\
Assault & 4 & 4 & 8 \\
Firearms & 0 & 1 & 1 \\
Road traffic accident & 2 & 3 & 5 \\
Non-accidental injury & 3 & 2 & 5 \\
Total number & 54 & 42 & 96 \\
\hline
\end{tabular}


Table III. Grade I injuries $(n=54)$ : pre- and post-operative visual acuity according to age

\begin{tabular}{|c|c|c|c|c|c|c|}
\hline & NA & $6 / 6$ & $6 / 9-6 / 18$ & $6 / 24-6 / 60$ & $<6 / 60$ & Total \\
\hline \multicolumn{7}{|c|}{ Pre-operative VA } \\
\hline $0-8$ years & $13(24 \%)$ & $1(2 \%)$ & $5(9 \%)$ & $3(6 \%)$ & $5(11 \%)$ & 27 \\
\hline $9-16$ years & 0 & $5(9 \%)$ & $12(22 \%)$ & $3(6 \%)$ & $7(13 \%)$ & 27 \\
\hline Total & $13(24 \%)$ & $6(11 \%)$ & $18(33 \%)$ & $4(7 \%)$ & $13(24 \%)$ & 54 \\
\hline \multicolumn{7}{|c|}{ Post-operative VA } \\
\hline $0-8$ years & 0 & $10(19 \%)$ & $9(17 \%)$ & $6(11 \%)$ & $2(4 \%)$ & 27 \\
\hline $9-16$ years & 0 & $17(31 \%)$ & $6(11 \%)$ & $4(7 \%)$ & 0 & 27 \\
\hline Total & 0 & $27(50 \%)$ & $14(26 \%)$ & $11(20 \%)$ & $2(4 \%)$ & 54 \\
\hline
\end{tabular}

NA, not able to record visual acuity.

repair. The average time for contact lens fitting was 7 months after the injury (range 6 weeks to 14 months). The 5 children who had an intraocular lens inserted were aged $10,11,11,13$ and 15 years and they achieved a final visual acuity of $6 / 9$ in 3 cases, $6 / 12$ in 1 case, and $6 / 60$ in 1 case due to cystoid macula oedema.

\section{DISCUSSION}

The approximate 4:1 ratio of boys to girls in this series corresponds closely with other series on ocular trauma. ${ }^{7}$ The male preponderance is more marked in injuries sustained in a domestic setting and in cases of assault. If the aetiology of the injury is compared with the visual outcome there is no correlation between the two and "sharp" lacerations from glass do no better than ragged lacerations due to fingers or sticks. It is of note that firearms tend to cause more severe ocular damage, with only one injury of this aetiology restricted to the anterior segment.

Grade 1 injuries (Table II) do well if the wound does not involve zone 1 and there is a careful repair with minimal post-operative astigmatism. In children less than 8 years old a visual acuity of $6 / 18$ or better was achieved in $70 \%$ $(n=19)$, but only $37 \%(n=10)$ achieved $6 / 6$ despite intensive orthoptic supervision. In the older age group $85 \%(n=23)$ achieved $6 / 18$ or better with $63 \%(n=17)$ achieving $6 / 6$ despite very similar injuries. This highlights how even a small optical change corrected with spectacles and then occlusion can fail to bring many of these cases back to 6/6. An intolerance and unwillingness to wear the spectacles was recorded in some of these case notes.

Grade 1 injuries that involve zone $1(n=18)$ tend to have a worse outcome with only $55 \%(n=10)$ achieving a visual acuity better than $6 / 18$. The $44 \%(8 / 18)$ who did worse than $6 / 18$ had a mean cylinder of $3.5 \mathrm{DC}$; it may also be that the degree of scarring is worse, with some irregularity leading to greater difficulty with the optical correction. The mean cylinder of those with an injury in zone 2 was 1.5 DC and 26 of 34 achieved an acuity of 6/9. If the under-eights were further divided into $0-4$ years and 5-8 years it became apparent that those with the grade 1 injuries that do worse are in the younger age group. It has previously been well demonstrated that the amount of residual astigmatism varies with the length of the laceration.

Eagling ${ }^{4}$ commented in 1976 on the poor visual results in grade 2 injury in children under 8 years of age. This was ascribed to amblyopia, problems in achieving clear media and adequate optical correction within 2 months of injury. Despite advances in contact lens materials, speed of application of the lens following injury and improved surgical instrumentation there has been little improvement in the visual outcome achieved at the Birmingham and Midland Eye Hospital.

Grade 2 injury in younger children creates considerable difficulties in deciding upon appropriate management of aphakia. Contact lenses, although reported to have up to an $80 \%$ acceptance rate in some units, ${ }^{8}$ were found to be poorly tolerated in this group, accounting for the relatively poor results. It is of note that the average length of time for fitting was 12 days. Intraocular lens implantation may have a role and several groups have tried this. ${ }^{9.10}$ However, there is a risk of severe uveitis and some feel this may not justify the use of intraocular lenses in this population. ${ }^{9}$ Epikeratophakia provides an alternative ${ }^{\prime \prime}$ but it has not been as successful as hoped. In the under-eight age group results were poor due to amblyopia, with only $55 \%$ $(n=10)$ of the children achieving a visual acuity of $6 / 18$ or better; 6 achieved $6 / 60$ or worse due to amblyopia despite early optical correction and intensive orthoptic supervision. The problems associated with contact lens

Table IV. Grade 2 injuries $(n=42)$ : pre- and post-operative visual acuity according to age

\begin{tabular}{|c|c|c|c|c|c|c|}
\hline & $\mathrm{NA}$ & $6 / 6$ & $6 / 9-6 / 18$ & $6 / 24-6 / 60$ & $<6 / 60$ & Total \\
\hline \multicolumn{7}{|c|}{ Pre-operative VA } \\
\hline $0-8$ years & $10(24 \%)$ & 0 & $1(2 \%)$ & $1(2 \%)$ & $6(14 \%)$ & 18 \\
\hline Total & $10(24 \%)$ & 0 & $2(4 \%)$ & $3(7 \%)$ & $27(64 \%)$ & 42 \\
\hline \multicolumn{7}{|c|}{ Post-operative VA } \\
\hline $0-8$ years & 0 & 0 & $10(24 \%)$ & $2(5 \%)$ & $6(14 \%)$ & 18 \\
\hline Total & 0 & $3(7 \%)$ & $26(62 \%)$ & $6(15 \%)$ & $7(17 \%)$ & 42 \\
\hline
\end{tabular}

NA, not able to record visual acuity. 
correction following surgery for congenital cataracts are well documented and trauma cases appear to do equally poorly in this younger age group. Only 3 children had an intraocular lens implant and this precludes judgement as to their performance, although 2 of them did well.

Six of the children underwent primary lens extraction while 6 underwent a secondary procedure; no visual difference between these two groups was found. Two further patients had their lens extracted after a progression of the opacity and received an intraocular lens; they achieved a visual acuity of 6/9. In the patient who received an intraocular lens and developed cystoid macular oedema the lens was put in soon after injury. This would seem to indicate that intraocular lens insertion is better in the quiet eye, although in most cases with significant opacity it would be very amblyogenic to wait for this.

The time taken to commence the surgical procedures was highly variable and dependent on many factors. Often most of the delay was due to the time taken to attend hospital in the first instance, mostly because of a failure on the part of the parents to appreciate the severity of the wound. Those presenting later after injury did not tend to do worse than those presenting early; in these the wound was often plugged by iris.

The pre-operative visual acuity in grade 1 injury when it was good was a reliable indicator of a good result. If it was poor it was a poor indicator of outcome. In grade 2 injury pre-operative acuity was not a reliable indicator of result.

Of the 96 patients reviewed in this study $14(15 \%)$ failed to achieve 6 months of follow-up. Five of these were discharged as they achieved $6 / 9$ or bettcr, were over 8 years old and there was no significant astigmatism or lens damage.

The other cases were followed until they were 10 years old. They were seen at least once a year and more frequently when there were any ocular sequelae: lens damage, central corneal damage or astigmatism to threaten the vision in the eye.

Nine patients failed to attend at any central or peripheral clinic and were lost to follow-up despite several appointments being sent and addresses checked. This may have been because the patient was seeing well and it was felt a review was not necessary. However, although this is an inner city hopsital and the figures for failing to come for review may seem low, it could be through lack of initial education and explanation, with the parents not understanding the need for further review and treatment. It is possible an education sheet should be provided on admission and re-emphasised before discharge to improve follow-up. If these patients had been reviewed the overall visual results obtained may have been better, as the last recorded acuity was used in this study.

It is interesting that none of these cases developed an endophthalmitis despite some cases having a very long delay in presentation. This may be due to the inherent antibacterial activity of the aqueous as well as the rush of aqueous fluid that will occur at the time of injury. However, one 9-year-old girl developed severe inflammation in her other eye within 2 weeks of the injury, having had a prolapse of iris; this episode of presumed sympathetic ophthalmitis was treated with high-dose topical and systemic steroids and resolved. It has subsequently remained quiet. None of the patients had developed corneal decompensation, but follow-up over a number of years may reveal some cases coming to light.

Good prognostic factors that were identified are: Age over 8 years; pre-operative visual acuity, if good; zone 2 injury; lens remaining intact; low level of astigmatism even in central wounds; perforation of less than onequarter corneal diameter; parental motivation if spectacles or contact lens are prescribed. Poor indicators of outcome are: mechanism of injury; length of time prior to surgery. Prevention, where possible, is better than cure and parents should remain vigilant to the many hazards even in a domestic setting.

We recognise that there are faults with the study: some grade 1 injuries may have been treated with a bandage contact lens only and not included in this study; the operations were performed by many different surgeons; the length of wound was not recorded; it is a retrospective survey and therefore relies on the accuracy of the notes; preoperative visual acuity was mostly performed by medical staff in casualty with no attempt at a more accurate visual acuity pre-operatively; post-operatively visual acuity was assessed in the orthoptic department and in many cases there was no record of stereopsis.

We would like to thank the Consultants at the Birmingham and Midland Eye Hospital for allowing their cases to be presented and discussed.

Key words: Child ren, Ocular injury. Penetrating, Prognosis.

\section{REFERENCES}

1. Parver LM. Eye trauma: the neglected disorder. Arch Ophthalmol 1986;104:1452-3.

2. Rapoport I, Romen M, Kinek $\mathrm{M}$ et al. Eye injuries in children in Israel. Arch Ophthalmol 1990;108:376-9.

3. Strahlman E, Elman M, Daub E, Baker S. Causes of paediatric eye injuries. Arch Ophthalmol 1990;108:603-6.

4. Eagling EM. Perforating injuries of the eye. Br J Ophthalmol 1976:60:732-6.

5. de Juan E, Stenberg P, Michels RG. Penetrating ocular injuries: types of injury and visual results. Ophthalmology 1990; 108:603-6.

6. Luff AJ, Hodgkins PR, Baxter RJ, Morrell AJ, Calder I. Aetiology of perforating eye injury. Arch Dis Child 1993; 68:682-3.

7. Strahlman E, Elman M, Daub E, Baker S. Causes of paediatric eye injury. Arch Ophthalmol 1990;108:603-6.

8. Epstein RJ, Fernandez A, Gammon JA. The correction of aphakia in infants with hydrogel extended wear contact lenses. Ophthalmology 1988;95:1102-6.

9. Taylor D. Monocular infantile cataract, intraocular lenses and amblyopia. Br J Ophthalmol 1989;73:857-8.

10. Burke JP, Willshaw HE, Young JDH. Intra-ocular lens implants for uniocular cataracts in children. $\mathrm{Br} \mathrm{J}$ Ophthalmol 1989;73:860-4.

11. Morgan KS, McDonald MB. Hiles DA, et al. The nationwide study of epikeratophakia for aphakia in older children. Ophthalmology 1988;95:526-31. 